\title{
Serum Ferritin As A Novel Predictive Marker For Breast Cancer Metastasis And Correlation With Metastasis-Associated Burden
}

\section{Xufeng Yao}

The First Affiliated Hospital of Chongqing Medical University

Xiang $\mathrm{He}$

The First Affiliated Hospital of Chongqing Medical University

\section{Lu Gan}

The First Affiliated Hospital of Chongqing Medical University

\section{Xiaoyi Wang}

The First Affiliated Hospital of Chongqing Medical University

Qiao Cheng ( $\nabla$ chengqiao@hospital.cqmu.edu.cn )

The First Affiliated Hospital of Chongqing Medical University

\section{Research Article}

Keywords:

Posted Date: March 3rd, 2022

DOI: https://doi.org/10.21203/rs.3.rs-1320044/v1

License: (c) (1) This work is licensed under a Creative Commons Attribution 4.0 International License. Read Full License 


\section{Abstract}

The present study evaluated the potential of serum ferritin to predict the breast cancer metastasis incidence and correlation with the metastasis-associated burden. A total of 364 patients with breast cancer comprising 123 patients with distant metastasis and 241 with no metastasis were retrospectively reviewed. The levels of the three serum tumor markers, namely ferritin, CA15-3, and carcinoembryonic antigen (CEA), were analyzed in different pathological types and metastatic sites. Of these, serum ferritin was associated with breast cancer metastasis with the highest sensitivity and specificity. An optimal ferritin cut-off value of $170.7 \mu \mathrm{g} / \mathrm{mL}$ was obtained.

The capacity for metastatic breast cancer detection was significantly higher when using a combination of two or all three markers for detection. The combination of ferritin and CA15-3 could be the optimal choice considering socioeconomics and predictive ability. Ferritin has higher predictive efficiency in HR- / HER2+ and HR- / HER2- molecular subtypes. The baseline serum ferritin, CA15-3, and CEA levels in breast cancer reflected the extent of tumor dissemination to the metastatic sites, which reflected the correlation between tumor markers and metastasis-associated burden. The serum ferritin, CA15-3, and CEA levels in patients with liver metastasis were significantly higher than those in patients with lung and bone metastasis. Therefore, ferritin can be a noninvasive biomarker for monitoring breast cancer metastasis.

\section{Introduction}

In 2020, breast cancer accounted for 2.26 million new cases and 0.68 million deaths globally, whereas lung cancer accounted for more than 2.2 million cases. Breast cancer has replaced lung cancer as the most common cancer in the world. Additionally, 0.42 million new breast cancer cases were observed among Chinese women, reaching $19.9 \%$ of all new cancer cases[1]. Several patients exhibited distant metastasis at the time of primary treatment. Additionally, some patients exhibited recurrence and metastasis after systematic treatment[2]. Early detection of metastatic lesions is vital for improving survival, making the development of accurate and easy monitoring methods is crucial. Clinical research on breast cancer including the detection of circulating tumor markers, circulation of tumor cells, and circulation of tumor DNA has been focused upon[3]. Presently, the application of serum tumor markers for breast cancer is based on the 2007 edition of American Society of Clinical Oncology (ASCO) guidelines [4], which recognizes the value of circulating tumor markers, including the three serum tumor markers, CA15-3, carcinoembryonic antigen (CEA), and ca27.29, for monitoring some metastatic breast cancers. In most hospitals in China, CA15-3 and CEA are used as breast cancer markers despite their limited specificity and sensitivity.

More specific and sensitive cost-effective tumor markers must be identified to predict distant metastasis of breast cancer. Ferritin may be clinically useful as a biomarker for predicting distant metastasis of breast cancer. Studies have exhibited the wide distribution of serum ferritin in several types of tissue cells, which is mainly involved in iron metabolism. Additionally, several solid malignant tumors can synthesize and secrete ferritin[5-8]. A study monitoring seven biochemical parameters, namely serum ferritin, C- 
reactive protein, CEA, acid glycoprotein, total alkaline phosphatase, sialyltransferase, and urinary hydroxyproline

/creatinine ratio in 17 patients with metastatic breast cancer exhibited the highest increase in serum ferritin in patients with metastatic breast cancer, reaching 88\% (15 / 17). Bezwoda et al. [9] exhibited that high serum ferritin levels ( $>400 \mu \mathrm{g} / \mathrm{L}$ ) were often associated with liver metastasis of breast cancer. A retrospective study by Williams et al. [10] exhibited that the serum ferritin level in patients with lung metastasis was significantly lower than that in patients with other metastases. However, these studies had a small sample size[11]. Ferritin did not increase in non-metastatic breast cancer, which is inconsistent with existing reports[12]. Additionally, ferritin must be compared and combined with current breast tumor markers such as CA15-3 and CEA to predict the recurrence and metastasis of breast cancer.

The present study reported the results of patients with breast cancer who exhibited distant metastasis. The present study was performed to evaluate the usefulness of the serial assessment of serum ferritin combined with CEA and CA15-3 for prediction of symptomatic metastasis in patients with primary breast cancer and incidence and intensity of breast cancer metastasis, which is valuable for providing more data about the usage of serum markers including ferritin.

Patients and methods

This study was approved by the Institutional Review Board of the First Affiliated Hospital of Chongqing Medical University and conformed to the ethical standards of the Declaration of Helsinki. The patients or their family members provided written informed consent for each follow-up as required. Data were collected by the electronic medical records system at our institute. The present study retrospectively reviewed 364 patients with breast cancer between June 2018 and December 2020 in our institution. The patients were divided into 123 cases of distant metastasis and 241 cases of non-metastasis by imaging and pathological diagnosis. Immunohistochemistry, pathological results, tumor marker detection results, and other data such as demographic characteristics and clinical data were investigated between the two groups. Serum tumor markers were measured by enzyme-linked immunosorbent assay using TECAN and reagent kits (Sweden IDL Biotech).

\section{Statistical analysis}

The receiver operating characteristics (ROC) curve was analyzed to determine the critical value, sensitivity, and specificity of independent factors or the combination for predicting metastasis. One-way analysis of variance was utilized to compare the relationship between baseline serum ferritin, CEA, CA153 , and metastasis positive results. Comparison of energy efficiency between ferritin combined with CA153 and any other tumor markers in predicting breast cancer metastasis. SPSS 26.0 statistical software was used to analyze the data.

\section{Results}




\section{Patient characteristics}

The average age of patients with metastatic breast cancer and those with no metastasis was $50.9 \pm 11.0$ years and $48.8 \pm 10.8$ years, respectively. Among 123 patients with metastatic breast cancer, 15 (12.20\%) patients were pathologically diagnosed with HR+/HER2+, 50 (40.65\%) were diagnosed with HR+/HER2-, $30(24.39 \%)$ were diagnosed with HR-/HER2+, and 28 (22.76\%) were diagnosed with HR-/HER2-. Of the 241 enrolled patients with non-metastatic breast cancer, 47 (19.50\%) patients were pathologically diagnosed with HR+/HER2+, 90 (37.35\%) were diagnosed with HR+/HER2-, 53 (21.99\%) were diagnosed with HR-/HER2+, and 51 (21.16\%) were diagnosed with HR-/HER2-. The clinicopathological characteristics of the 364 patients are presented in Tables 1 and Table 2.

\section{Table 1: General characteristics of study population.}

\section{Table 2: General characteristics of study population.}

High serum ferritin levels are associated with metastasis of breast cancer

The serum ferritin, CA15-3, and CEA levels in the metastatic breast cancer group were significantly higher than those in the non-metastatic group $(p<0.05)$. ROC analysis was performed to evaluate the predictive effect of serum ferritin, CA15-3, and CEA on breast cancer metastasis. Area under the curve (AUC) and cut-off value were calculated. The AUC of serum ferritin, CA15-3, and CEA was 0.869 (Cl: 0.837-0.902; P $<0.001$ ), 0.799 (Cl: 0.758-0.840; $\mathrm{P}<0.001$ ), and 0.759 (Cl: 0.712-0.805; $\mathrm{P}<0.001$ ), respectively (Fig. 1). The AUC of the combinations of serum ferritin and CA15-3; serum ferritin and CEA; CA15-3 and CEA; and serum ferritin, CA15-3, and CEA was 0.903 (Cl: 0.875-0.931; $\mathrm{P}<0.001), 0.894$ (Cl: 0.865-0.924; $\mathrm{P}<$ $0.001), 0.813$ (Cl: 0.775-0.854; $\mathrm{P}<0.001)$, and 0.909 (Cl: 0.882-0.936; $\mathrm{P}<0.001)$, respectively (Fig. 1).

The best cut-off values of tumor markers were obtained by calculating the maximum Youdon index. The optimal cut-off value of serum ferritin was $170.7 \mu \mathrm{g} / \mathrm{mL}$ with a sensitivity of $71.37 \%$ and specificity of 88.32\%. The CA15-3 cut-off value was $21.2 \mathrm{U} / \mathrm{mL}$ with a sensitivity and specificity of $56.74 \%$ and $91.94 \%$, respectively, whereas that for CEA was $2.79 \mu \mathrm{g} / \mathrm{mL}$ with a sensitivity and specificity of $66.05 \%$ and $82.85 \%$, respectively. The combination of serum ferritin and CA15-3 resulted in a sensitivity and specificity of $74.88 \%$ and $92.67 \%$, respectively. The combination of serum ferritin and CEA resulted in a sensitivity and specificity of $81.86 \%$ and $84.67 \%$, respectively. The combination of CA15-3 and CEA resulted in a sensitivity and specificity of $55.87 \%$ and $94.51 \%$, respectively. The combination of all three markers resulted in a sensitivity and specificity of $85.45 \%$ and $83.88 \%$, respectively for prediction of breast cancer metastasis. Thus, the capacity for detection metastatic breast cancer is significantly higher when using a combination of two or all three markers for detection. Among the combined two markers, combined serum ferritin and CA15-3 exhibited the highest AUC value, which was not significantly different from the combined three markers. Therefore, the combination of serum ferritin and CA15-3 was the optimal choice from the perspective of socioeconomics and predictive ability. 
Table3 Ferritin, CA153 cancer antigen 15-3, CEA carcinoembryonic antigen P25, the 25th percentile; P75, the 75th percentile.

High serum ferritin exhibits the highest predictive value in HR-/HER2 + and HR-/HER2- subtypes of breast cancer

Patients were divided into four types to evaluate the predictive value of serum ferritin, CA15-3, and CEA for breast cancer with different subtypes, and the AUCs of individual markers for patients with HR+/HER2 + breast cancer were measured (Fig. 2). The AUC of serum ferritin, CA15-3, and CEA was 0.641 (Cl: 0.516$0.765 ; \mathrm{P}=0.036), 0.742$ (Cl: $0.623-0.861 ; \mathrm{P}<0.001), 0.711$ (Cl: $0.576-0.845 ; \mathrm{P}=0.002$ ), respectively. For patients with HR+/HER2- breast cancer, the AUC of serum ferritin, CA15-3, and CEA was 0.869 (Cl: $0.821-$ 0.918; $\mathrm{P}<0.001), 0.894$ (Cl: 0.851-0.938; $\mathrm{P}<0.001), 0.808$ (Cl: 0.734-0.882; $\mathrm{P}<0.001$ ), respectively. For patients with HR-/HER2 + breast cancer, the value was 0.846 (Cl: $0.770-0.923 ; \mathrm{P}<0.001), 0.722$ (Cl: $0.627-0.817 ; \mathrm{P}<0.001), 0.755$ (Cl: 0.662-0.847; $\mathrm{P}<0.001$ ), respectively. For patients with HR-/HER2breast cancer, the value was 0.845 (Cl: $0.779-0.910 ; \mathrm{P}<0.001), 0.662$ (Cl: $0.558-0.765 ; \mathrm{P}=0.002), 0.661$ (Cl: $0.561-0.761 ; P=0.002)$, respectively. CA15-3 exhibited the highest predictive value in patients with $\mathrm{HR}+/ \mathrm{HER} 2+$ and HR+/HER2 - breast cancer. Serum ferritin exhibited the highest predictive value in patients with HR-/HER2 + and HR-/HER2- breast cancer.

\section{High serum ferritin is associated with increased metastasis- associated tumor burden}

Subgroup analysis was performed to evaluate the relationship between serum ferritin, CA15-3, CEA levels, and metastasis to the lymph nodes and distant organs. In patients without distant metastasis, baseline serum ferritin, CA15-3, and CEA levels increased as the number of metastatic lymph nodes increased. Ferritin $(P<0.001)$ and CEA $(P=0.001)$ levels were higher in patients with more than three metastatic lymph nodes than those with 1-2 or no metastatic lymph nodes. In patients with distant metastasis, ferritin $(P=0.026)$ and CA15-3 $(P<0.001)$ levels were higher in patients exhibiting metastasis to more than three distant organs than in those exhibiting 1-2 or no distant organ metastases (Fig. 3) (Table 4). Patients were divided into non-metastasis, single distant metastasis, and multiple distant metastasis groups. Serum ferritin, CA15-3, and CEA levels in the multiple metastasis group were significantly higher than those in the other two groups. No significant difference between these levels was observed between non-metastasis and single distant metastasis groups. ROC analysis exhibited that the AUC of ferritin, CA15-3, and CEA among the single distant metastasis group was 0.765 (Cl: $0.712-0.818 ; \mathrm{P}<0.001$ ), 0.677 (Cl: $0.615-0.739 ; P<0.001), 0.576$ (Cl: 0.505-0.647; $P=0.021$ ), respectively. Among the multiple distant metastasis group, the AUC of ferritin, CA15-3, and CEA was 0.868 (Cl: $0.830-0.907 ; \mathrm{P}<0.001$ ), 0.855 (Cl: $0.809-0.902 ; \mathrm{P}<0.001$ ), and 0.881 (Cl: $0.838-0.924 ; \mathrm{P}<0.001$ ), respectively, indicating the higher sensitivity and specificity of serum ferritin, CA15-3, and CEA levels in predicting metastasis in the multiple metastasis group (Fig. 4).

High serum ferritin exhibits the greatest predictive value for liver and bone metastasis 
Patients with breast cancer with distant metastasis were grouped according to various metastasis sites. The levels of serum tumor markers in patients with liver metastasis were significantly higher than those with metastasis to other organs (Table 3). The differences in ferritin and CEA values in each group were statistically significant $(P<0.001)$. ROC analysis exhibited that these serum tumor markers are more sensitive and specific for prediction of liver and bone metastasis, especially liver metastasis. The predictive value of serum tumor marker levels in patients with lung metastasis was significantly lower than that in patients with other metastases (Fig. 5 ).

\section{Discussion}

While the prognosis of patients with breast cancer has significantly improved due to the progressive treatment, $30 \%$ of early-stage breast cancer still remains metastatic, which remains the leading cause of death in breast cancer [13]. Therefore, effective and cheap tumor markers must be selected from the various available markers for the monitoring of patients with breast cancer, especially in developing countries with limited health budgets.

CA15-3 and CEA have been widely used clinically as the breast tumor markers for monitoring metastatic breast cancer as recommended by 2007 ASCO guidelines. Most breast physicians have chosen to routinely detect these two markers for patients with breast cancer during and after the therapy. The present study analyzed the serum ferritin, CEA, and CA15-3 independently and in combination for the diagnosis of metastatic breast cancer, and their sensitivity and specificity in predicting metastasis to reveal their efficacy in predicting breast cancer metastasis. We found that serum ferritin is a more efficient prognostic marker for metastatic disease than the traditional breast cancer markers CA15-3 and CEA. Additionally, the cut-off value of each metastatic marker was calculated by comparing the maximum Youdon index. The cut-off value of serum ferritin was $170.7 \mu \mathrm{g} / \mathrm{mL}$ with a sensitivity of $71.37 \%$ and specificity of $88.32 \%$. The CA15-3 cut-off value was $21.2 \mathrm{U} / \mathrm{mL}$ with a sensitivity of $56.74 \%$ and specificity of $91.94 \%$, whereas that for CEA was $2.79 \mu \mathrm{g} / \mathrm{mL}$ with a sensitivity of $66.05 \%$ and specificity of $82.85 \%$.

Stieber et al. [14] observed that the sensitivities of CEA, CA15-3, and combination of both in metastatic breast cancer diagnosis were $40.6 \%, 55.6 \%$, and $66.3 \%$, respectively. CA15-3 is superior to CEA as a tumor marker in metastatic breast cancer diagnosis with higher sensitivity and specificity [15]. This finding is concurrent with that of other studies. Additionally, ferritin is effective for prediction of breast cancer metastasis with the highest sensitivity $(71.37 \%)$ and specificity $(88.32 \%)$ among the three markers.

However, a single tumor marker might not be the optimal choice when compared with the combination of markers. When using a combination of two or all three markers for detection, the capacity for metastatic breast cancer diagnosis is significantly higher. Additionally, the AUC of the serum ferritin and CA15-3 combination was 0.903, higher than those for the serum ferritin and CEA (0.894), and CA15-3 and CEA combinations (0.814). The highest AUC was 0.909 from the combination of serum ferritin, CA15-3, and CEA. The combination of all three markers resulted in the highest sensitivity of $85.45 \%$. However, the triple 
combination did not achieve the highest specificity. Several tumor markers do not require to be combined to improve prognostic sensitivity and specificity. Different markers have different sensitive parts, and too much inspection can lead to prediction error. The AUC of serum ferritin and CA15-3 combination was similar with that of the triple combination. Thus, the combination of serum ferritin and CA15-3 is the optimal choice socioeconomically.

The predictive value of the markers in breast cancer patients with different subtypes was analyzed to demonstrate the relation of the predictive efficiency to the molecular subtypes of breast cancer. The present study observed that serum ferritin exhibited the highest AUC in the HR-/HER2 + and HR-/HER2groups, whereas CA15-3 exhibited the highest AUC in the HR+/HER2 + and HR+/HER2- groups. Thus, the corresponding markers must be selected according to molecular typing results or the combination of serum ferritin and CA15-3 should be co-analyzed for metastasis monitoring.

Breast cancer metastasizes to the bone, lung, liver, and soft tissue most frequently [16]. Different tumor marker expression may relate to the varying metastatic sites and metastatic tumor burden. The present study observed that the values of serum ferritin, CA15-3, and CEA were associated with the numbers of metastatic lymph nodes and metastatic organs.

Yasasever $V$ et al. [17] observed that CA15-3 and CEA were elevated in breast cancer patients with bone metastases. Nicolini A exhibited that CEA, TPA, and CA15-3 were useful for the diagnosis of breast cancer bone metastases [18]. Lee JS et al. and Nicolini A et al. [19-20] observed the efficacy of CA15-3 and TPS as markers for breast cancer liver metastases. The sensitivity and specificity of CA15-3 in the diagnosis of breast cancer with liver metastasis were $52 \%$ and $80.8 \%$, respectively [21]. However, Geraghty JG et al. [22] and Yerushalmi $R$ et al. [23] reported no relationship between levels of CA15-3, CEA, CA125, and metastasis sites.

Serum ferritin, CA15-3, and CEA exhibited a positive correlation with the burden of breast cancer metastasis. Additionally, serum ferritin, CA15-3, and CEA in patients with liver metastasis were significantly higher than those in patients with lung and bone metastasis $(P<0.001)$. ROC analysis exhibited the highest sensitivity and specificity of serum ferritin in predicting liver metastasis among liver, lung, and bone metastasis. Liver plays a vital role in iron metabolism [24], explaining the high sensitivity and specificity of ferritin in predicting liver metastasis of breast cancer. However, other imaging and pathological examinations are still needed in clinical application because multiple distant metastasis may lead to a general increase in the three markers.

The present study exhibited the critical role of serum ferritin in predicting breast cancer metastasis. Serum ferritin may be used with high specificity and sensitivity to predict distant metastasis of breast cancer. It has higher predictive value in specific molecular typing and metastasis site. Ferritin levels specifically reflect the metastasis-associated burden of breast cancer. The inclusion of routine analysis of serum ferritin levels in clinical examination after breast cancer treatment may help improve therapeutic decisions and patient survival. Moreover, the combination of serum ferritin and CA15-3 may be used to 
further improve the sensitivity and specificity in the prediction of distant metastasis of breast cancer, which will be the main research direction in our follow-up work.

\section{Declarations}

Data availability

All data generated or analysed during this study are included in this published article (and its Supplementary Information files).

Acknowledgement

We appreciate the linguistic assistance provided by TopEdit (www.topeditsci.com) during the preparation of this manuscript.

Funding

This work was supported by research grants provided by the Medical Research Project of Chongqing Science Technology Commission and Chongqing Municipal Health Commission

(2021MSXM333).

Author contributions

All authors have contributed to this manuscript. C.Q., W.X.Y. and G.L. conceived and designed the project; C.Q. and Y.X.F. obtained the data; Y.X.F. and H.X. analysed and interpreted the data; Y.X.F. and C.Q. wrote the paper. All the authors read and approved the final manuscript.

Competing interest

The authors declare no competing interests.

\section{References}

1. Sung H, Ferlay J, Siegel RL, et al. (2021). Global cancer statistics 2020: GLOBOCAN estimates of incidence and mortality worldwide for 36 cancers in 185 countries. CA Cancer J Clin. 71(3):209-249.

2. A.J. Redig, S.S. McAllister, Breast cancer as a systemic disease: a view of metastasis, J. Intern. Med. 274 (2013) 113-126.

3. Koessler T, Addeo A, Nouspikel T. Implementing circulating tumor DNA analysis in a clinical laboratory: A user manual. Adv Clin Chem. 2019;89:131-188.

4. L. Harris, H. Fritsche, R. Mennel et al., "American Society of Clinical Oncology 2007 update of recommendations for the use of tumor markers in breast cancer," Journal of Clinical Oncology, vol. 25, no. 33, pp. 5287-5312, 2007. 
5. Ferreira C, Bucchini D, Martin ME, et al. Early embryonic lethality of $\mathrm{H}$ ferritin gene deletion in mice. J. Biol. Chem. 2000 04;275(5):302 1-3024

6. Santambrogio P, Cozzi A, Levi S, et al.Human serum ferritin G-peptide is recognized by anti-L ferritin subunit antibodies and concanavalin. $\mathrm{Br} J$ Haematol, 1987: 65, 235-237.

7. Lukina EA, Levina, AA, Mokeeva RA, et al.The diagnostic significance of serum ferritin indices in patients with malignant and reactive histiocytosis. $\mathrm{Br} \mathrm{J}$ Haematol, 1993: 83, 326-329.

8. Cazzola M, Arosio P, Bellotti V, et a1. Immunological reactivity of serum ferritin in patients with malignancy.Tumori, 1985:71, 547-554.

9. Bezwoda W, Derman D, Bothwell T, et al. Significance of serum concentrations of carcinoembryonic antigen, ferritin and calcitonin in breast cancer. Cancer. 1981,48: 1623-1628.

10. Williams MR, Turkes A, Pearson D, et al. Serum ferritin as a marker of therapeutic response in stage III and IV breast cancer. Eur J Surg Oncol. 1990, 16:22-27.

11. Robertson JF, Pearson D, Price MR, et al. Prospective assessment of the role of five tumour markers in breast cancer. Cancer Immunol. Immunother. 1991;33(6):403-410.

12. Garcia, J.A.; Fernandez, D.T.; Alvarez, E.A.; Gonzalez, E.B.; Montes-Bayon, M.; Sanz-Medel A. Iron speciation, ferritin concentrations and Fe:ferritin ratios in different malignant breast cancer cell lines: On the search for cancer biomarkers. Metallomics 2016, 10, 1090-1096.

13. O'Shaughnessy J. Extending survival with chemotherapy in metastatic breast cancer. Oncologist. 2005;10(S3 Suppl 3):20-9.

14. P. Stieber, D. Nagel, I. Blankenburg, V. Heinemann, M. Untch, I. Bauerfeind, et al., Diagnostic efficacy of CA $15-3$ and CEA in the early detection of metastatic breast cancer-a retrospective analysis ofkinetics on743 breast cancer patients, Clin.Chim. Acta 448 (2005) 228-231

15. D.F. Hayes, V.R. Zurawski Jr., D.W. Kufe, Comparison of circulating CA15-3 and carcinoembryonic antigen levels in patients with breast cancer, J. Clin. Oncol. 4 (1986) 1542-1550

16. R. Kucera, O. Topolcan, O. Fiala, J. Kinkorova, V. Treska, I. Zedníková, et al., The role of TPS and TPA in the diagnostics of distant metastases, Anticancer Res. 36 (2016) 773-777.

17. V. Yasasever, M. Dinçer, H. Camlica, D. Karaloğlu, N. Dalay, Utility of CA $15-3$ and CEA in monitoring breast cancer patients with bone metastases: special emphasis on "spiking" phenomena, Clin. Biochem. 30 (1997) 53-56.

18. A. Nicolini, P. Ferrari, A. Sagripanti, A. Carpi, The role of tumour markers in predicting skeletal metastases in breast cancer patients with equivocal bone scintigraphy, Br. J. Cancer 79 (1999) 1443-1447

19. J.S. Lee, S. Park, J.M. Park, J.H. Cho, S.I. Kim, B.W. Park, Elevated levels of serum tumor markers CA $15-3$ and CEA are prognostic factors for diagnosis of metastatic breast cancers, Breast Cancer Res. Treat. 141 (2013) 477-484.]

20. A. Nicolini, A. Carpi, P. Ferrari, L. Anselmi, C. Spinelli, M. Conte, et al., The role of tumour markers in improving the accuracy of conventional chest X-ray and liver echography in the post-operative 
detection of thoracic and liver metastases from breast cancer, Br. J. Cancer 83 (2000) 1412-1417.

21. R. Cao, L.P. Wang, Serological diagnosis of liver metastasis in patients with breast cancer, Cancer Biol. Med. 9 (2012) 57-62.

22. J.G. Geraghty, E.C. Coveney, F. Sherry, N.J. O'Higgins, M.J. Duffy, CA 15 - 3 in patients with locoregional and metastatic breast carcinoma, Cancer 70 (1992) 2831-2834.

23. R. Yerushalmi, S. Tyldesley, H. Kennecke, C. Speers, R. Woods, B. Knight, et al., Tumor markers in metastatic breast cancer subtypes: frequency of elevation and correlation with outcome, Ann. Oncol. 23 (2012) 338-345.

24. Suzy V. Torti,1David H. Manz,1,2Bibbin T. Paul,1 Nicole Blanchette-Farra,1and Frank M. Torti3. Iron and Cancer, Annu. Rev. Nutr. 2018. 38:97-125

\section{Tables}

Table 1: General characteristics of study population.

$\begin{array}{lll}\text { Character All cases } \mathrm{n}(\%) \mathrm{N}=364 \quad \text { Metastasis group } \mathrm{n}(\%) \mathrm{N}=123 & \begin{array}{l}\text { Non-metastasis group } \\ \mathrm{n}(\%) \mathrm{N}=241\end{array}\end{array}$

\section{Age \years】}

\begin{tabular}{|c|c|c|c|}
\hline$₫ 50$ & $169(46.42)$ & $64(52.03)$ & $105(43.57)$ \\
\hline$\geq 50$ & 195(53.57) & $59(47.97)$ & $136(56.43)$ \\
\hline \multicolumn{4}{|l|}{ HR } \\
\hline Positive & 202(55.49) & $65(52.85)$ & $137(56.85)$ \\
\hline Negative & 162(44.51) & $58(47.15)$ & $104(43.15)$ \\
\hline \multicolumn{4}{|l|}{ Her-2 } \\
\hline Positive & 145(39.84) & 45(36.59ه & $100(41.49)$ \\
\hline Negative & $219(60.16)$ & 78(63.41) & $141(58.51)$ \\
\hline \multicolumn{4}{|l|}{ Subtype } \\
\hline HR+Her-2+ & $62(17.04)$ & $15(12.20)$ & $47(19.50)$ \\
\hline HR+Her-2- & $140(38.46)$ & $50(40.65)$ & $90(37.35)$ \\
\hline HR-Her-2+ & $83(22.80)$ & $30(24.39)$ & $53(21.99)$ \\
\hline HR-Her-2- & $79(21.70)$ & $28(22.76)$ & $51(21.16)$ \\
\hline
\end{tabular}

Table 2: General characteristics of study population. 


\begin{tabular}{lll} 
Character & Metastasis group N=123 & $\mathbf{n} \llbracket \%$ \\
\hline Single distant metastasis & 60 & \\
\hline Bone & 16 & 26.67 \\
\hline Liver & 14 & 23.33 \\
\hline Lung & 19 & 31.67 \\
\hline Other organs & 11 & 18.33 \\
\hline Multiple distant metastasis & 63 & \\
\hline Including bone & 46 & 73.02 \\
\hline Including liver & 30 & 47.62 \\
\hline Including lung & 35 & 55.56
\end{tabular}

Table 3 Ferritin, CA153 cancer antigen 15-3, CEA carcinoembryonic antigen P25, the 25th percentile; P75, the 75th percentile. 
N Ferritin CA153

CEA

\begin{tabular}{|c|c|c|c|c|}
\hline & & Median ( P25, P75) & $\begin{array}{l}\text { Median (P25, } \\
\text { P75) }\end{array}$ & $\begin{array}{l}\text { Median (P25, } \\
\text { P75) }\end{array}$ \\
\hline Non-metastasis $\square A l l \square$ & 241 & $76.57(44.94,132.28)$ & $\begin{array}{c}10.40(7.37, \\
15.48)\end{array}$ & $2.30)^{1.63(1.10,}$ \\
\hline $\begin{array}{l}\text { Non-metastasis } \\
(\mathrm{HR}+\mathrm{Her} 2+)\end{array}$ & 47 & $96.93(51.52,167.70)$ & $\begin{array}{c}12.38(8.00 \\
19.60)\end{array}$ & $\begin{array}{l}2.14(1.20 \\
3.19)\end{array}$ \\
\hline $\begin{array}{l}\text { Non-metastasis } \\
\text { (HR+Her2-) }\end{array}$ & 90 & $85.20(42.51,184.52)$ & $\begin{array}{c}10.10(7.27, \\
16.05)\end{array}$ & )$^{1.72(1.22,2.52}$ \\
\hline $\begin{array}{l}\text { Non-metastasis (HR- } \\
\text { Her2+) }\end{array}$ & 53 & $86.10(56.56,139.75)$ & $\begin{array}{r}10.90(7.60, \\
15.80)\end{array}$ & $\begin{array}{l}1.70(1.20, \\
2.63)\end{array}$ \\
\hline $\begin{array}{l}\text { Non-metastasis (HR- } \\
\text { Her2-) }\end{array}$ & 51 & $93.14(46.30,166.55)$ & $2.70(8.74,18.18$ & $\begin{array}{l}1.49 \\
2.39)\end{array}(1.00$ \\
\hline Metastasis $\square A l \mid \square$ & 174 & )$^{307.80(151.22,600.05}$ & $\begin{array}{c}27.10(12.95 \\
157.25)\end{array}$ & $\begin{array}{r}5.00(1.80, \\
37.66)\end{array}$ \\
\hline Metastasis $\square \mathrm{HR}+\mathrm{Her} 2+\square$ & 26 & $138.15(69.14,356.75)$ & $\begin{array}{l}24.25(14.62, \\
42.92)\end{array}$ & $\begin{array}{l}4.16(1.93, \\
55.09)\end{array}$ \\
\hline Metastasis $\square$ HR+Her2-口 & 69 & )$^{394.50(216.35,785.56}$ & $\begin{array}{c}76.10(18.13 \\
700.00)\end{array}$ & $\left.\begin{array}{l}8.24 \\
49.99\end{array}\right)$ \\
\hline Metastasis $\square$ HR-Her2+口 & 40 & $295.30(152.68,590.35)$ & $\begin{array}{l}16.40(10.78, \\
65.66)\end{array}$ & $\left.\begin{array}{c}6.09 \\
22.12\end{array}\right)(1.58$ \\
\hline Metastasis $\square$ HR-Her2- - & 39 & )$^{268.27(164.70,402.90}$ & $\begin{array}{c}21.30(10.30, \\
111.45)\end{array}$ & $, 10.50)(1.34$ \\
\hline $\begin{array}{l}\text { Single distant } \\
\text { metastasis }\end{array}$ & 86 & )$^{192.45(122.96,352.63}$ & $\begin{array}{l}15.97(11.24, \\
29.18)\end{array}$ & $\begin{array}{l}2.08(1.18 \\
4.60)\end{array}$ \\
\hline $\begin{array}{l}\text { Multiple distant } \\
\text { metastasis }\end{array}$ & 88 & )$^{400.50(229.06,763.10}$ & $\begin{array}{l}81.76(20 . .67, \\
511.90)\end{array}$ & $\begin{array}{l}16.43(3.62, \\
50.71)\end{array}$ \\
\hline $\begin{array}{l}\text { Liver metastasis } \\
\text { [include! }\end{array}$ & 43 & $\begin{array}{l}515.70(246.95 \\
1050.50)\end{array}$ & $\begin{array}{l}111.45(39.35, \\
866.58)\end{array}$ & , 49.99$)^{21.01(4.45}$ \\
\hline $\begin{array}{l}\text { Bone metastasis } \\
\text { Dinclude }[\end{array}$ & 65 & )$^{367.15(203.82,637.80}$ & $\begin{array}{c}38.29 \\
286.91)\end{array}$ & $\left.\begin{array}{c}8.59 \\
45.38\end{array}\right)$ \\
\hline $\begin{array}{l}\text { Lung metastasis } \\
\text { Dincludel }\end{array}$ & 50 & )$^{365.64(170.85,619.35}$ & $\begin{array}{l}38.88(15.20 \\
484.00)\end{array}$ & $\begin{array}{l}5.10(1.68, \\
37.72)\end{array}$ \\
\hline
\end{tabular}

Table 4 Ferritin, CA153 cancer antigen 15-3, CEA carcinoembryonic antigen P25, the 25th percentile; P75, the 75th percentile. $P$ : values were calculated by Analysis of Variance. 


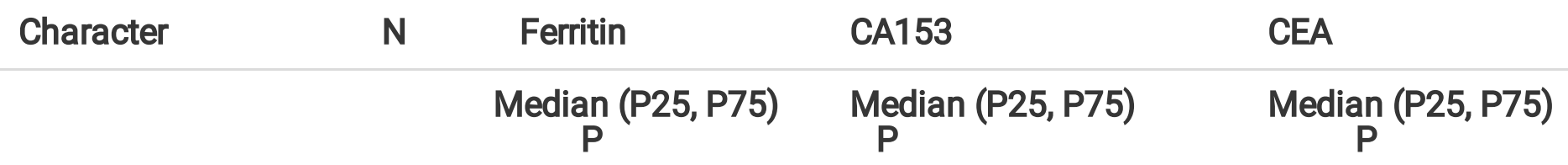

site

Non-metastasis group

241

$91.10(49.52$,

158.96)

Single-distant

86

$192.45(122.96$
$352.63)$
0.03

metastasis group

Multiple-distant

metastasis group
$88 \quad \begin{aligned} & 400.50 \\ & \quad 763.10) \\ & \end{aligned}$
$11.20(7.70,17.11)$

$1.70(1.20,2.60)$

$15.97(11.24,29.18)$

0.54

$2.08(1.18,4.60)$

0.868

\section{Figures}
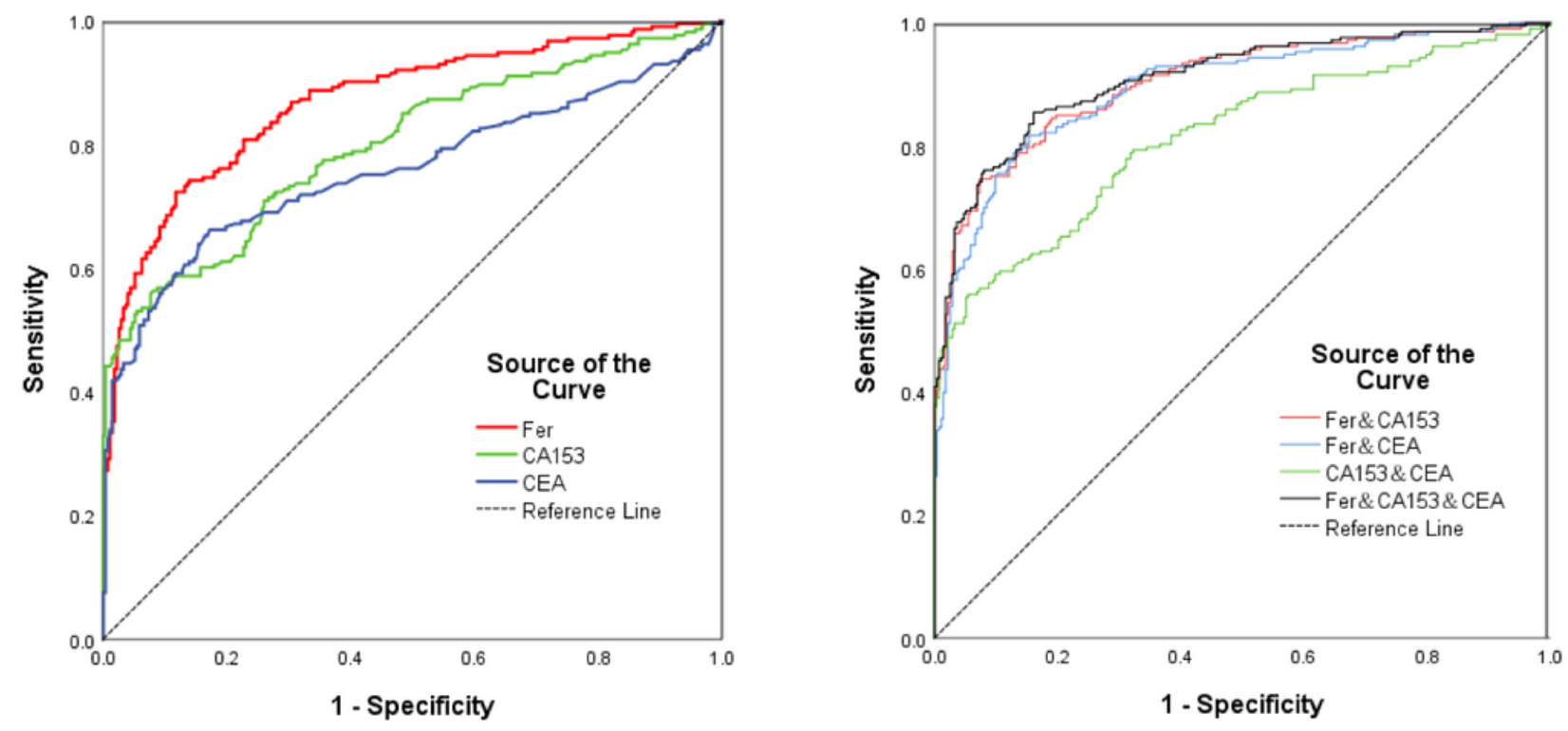

Figure 1

Three serum tumor markers were included in receiver operating curve (ROC) analyses for prediction of metastasis. Ferritin is valuable on prediction of breast cancer metastasis with the highest sensitivity and 
specificity among the 3 markers. When using a combination of two or all the three markers for detection, the capacity for detection metastatic breast cancer (MBC) is significantly higher.

HR+Her2+

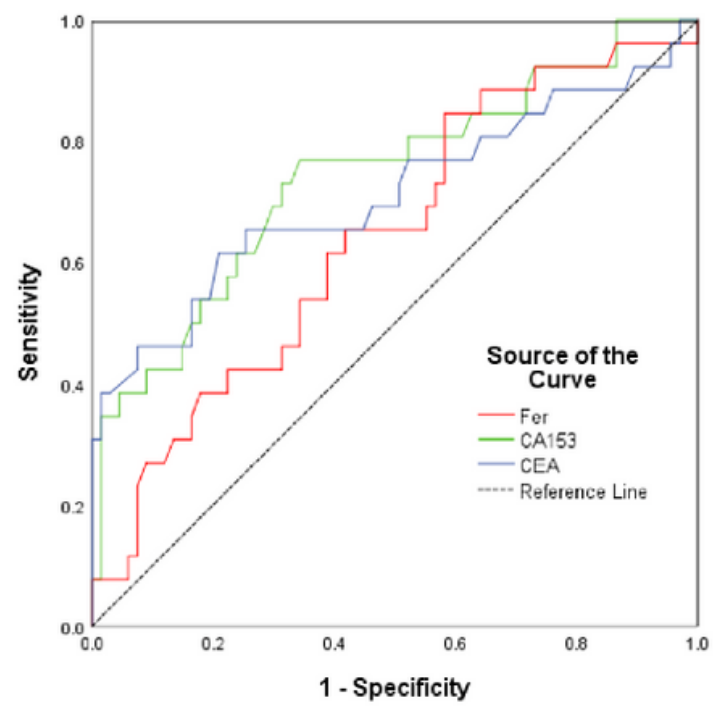

HR-Her2+

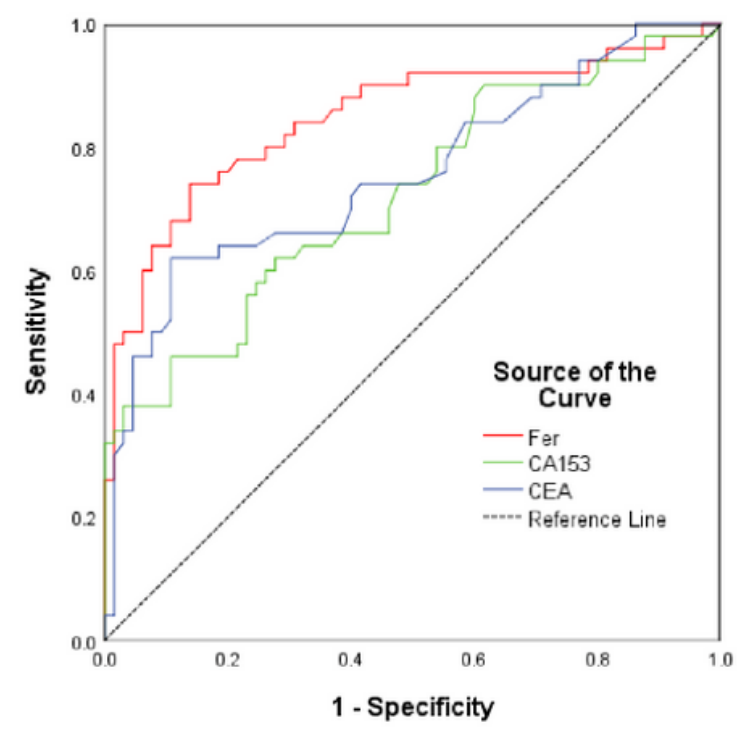

HR+Her2-

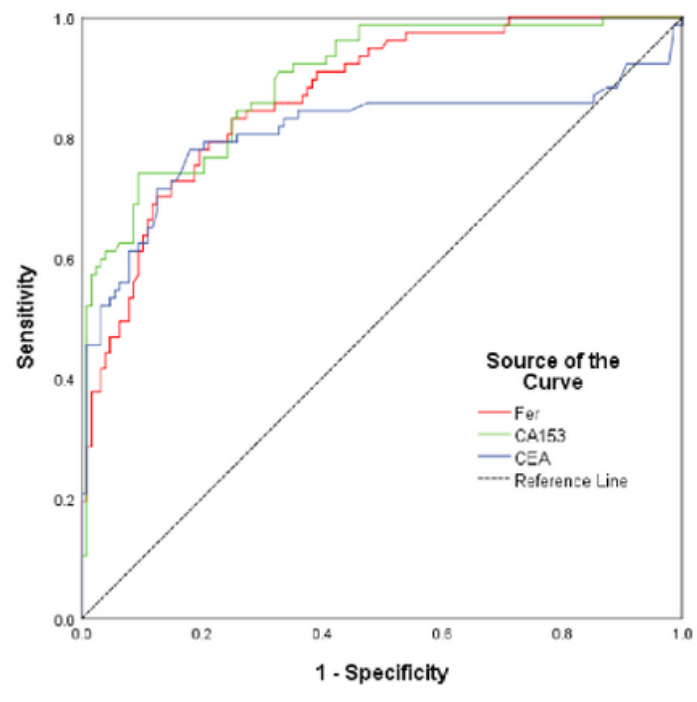

HR-Her2-

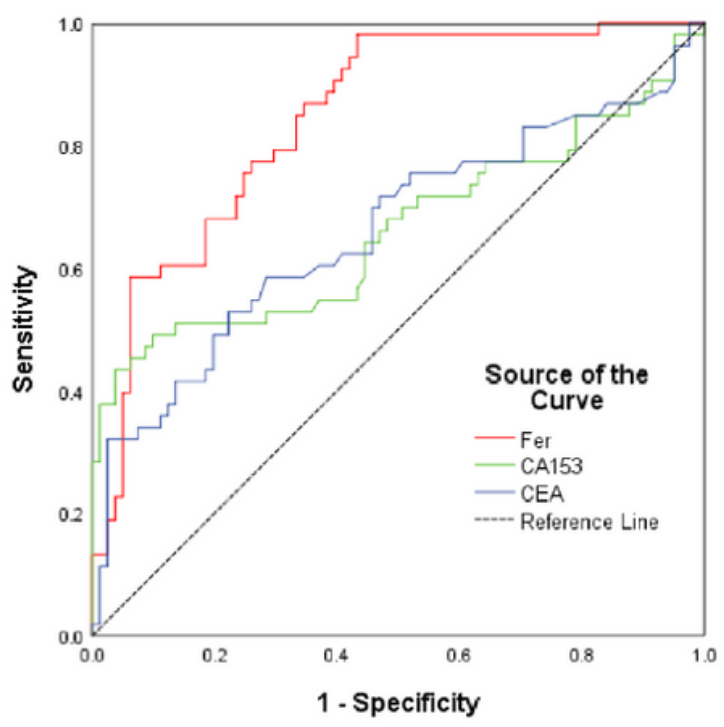

Figure 2

In different molecular subtypes, Receiver operating characteristic (ROC) curve analysis of different markers to predict the metastatic status. 

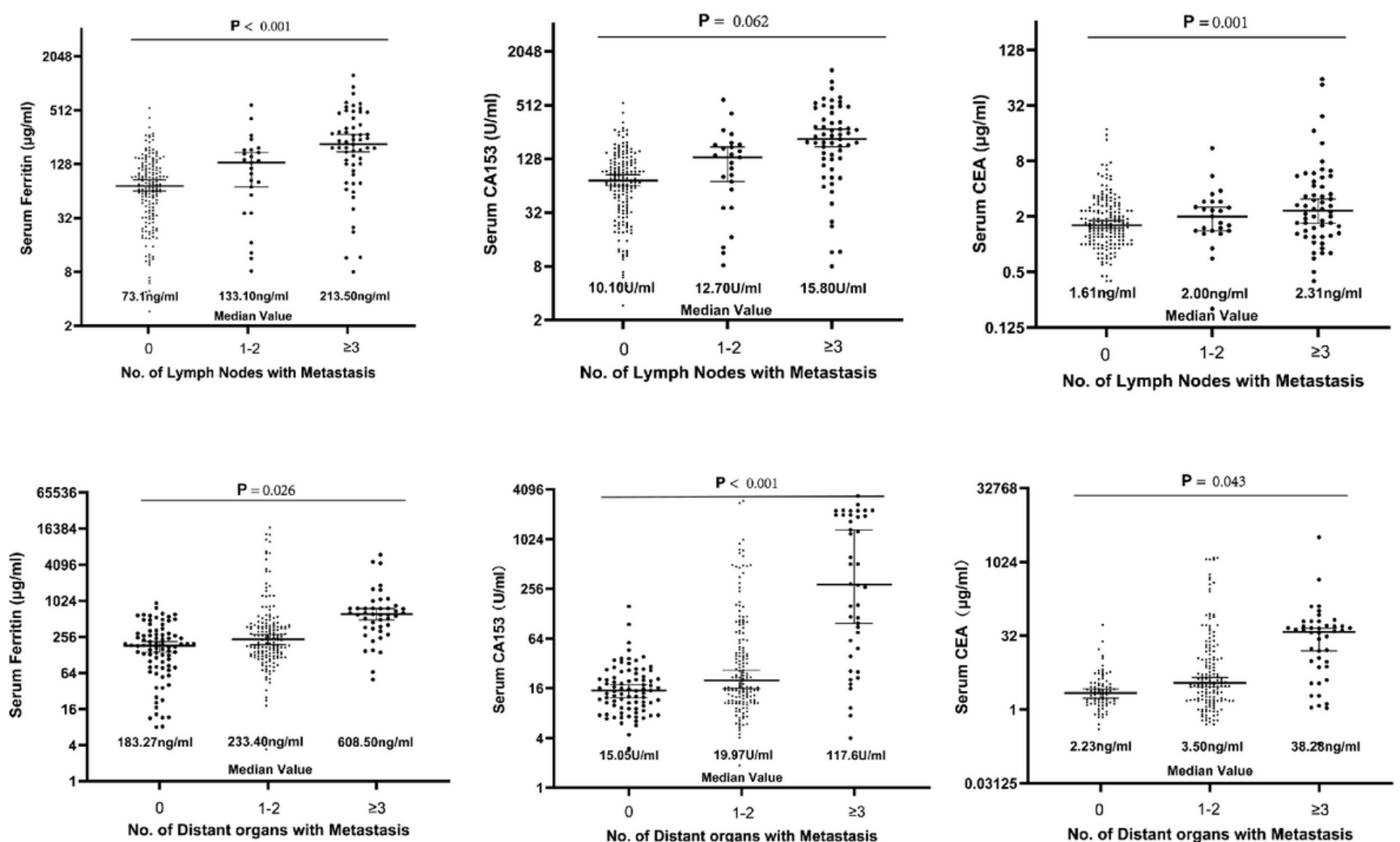

Figure 3

Baseline serum Ferritin, CA15-3, CEA (log2 scale on the y-axis) were significantly elevated with increasing burden of metastasis to lymph nodes and distant organs in MBC.

Single distant metastasis

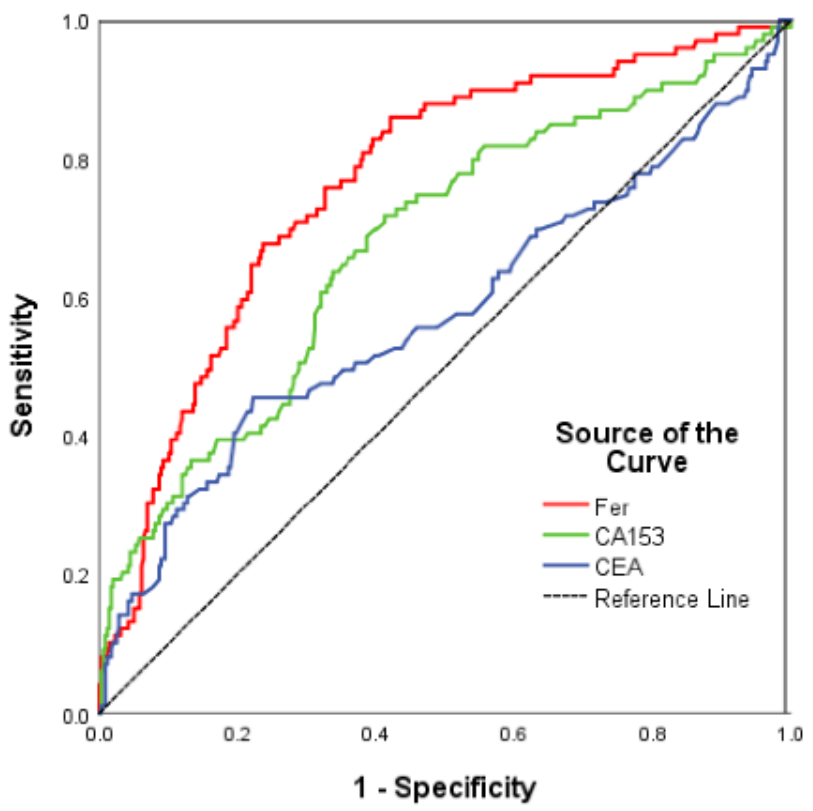

Multiple distant metastasis

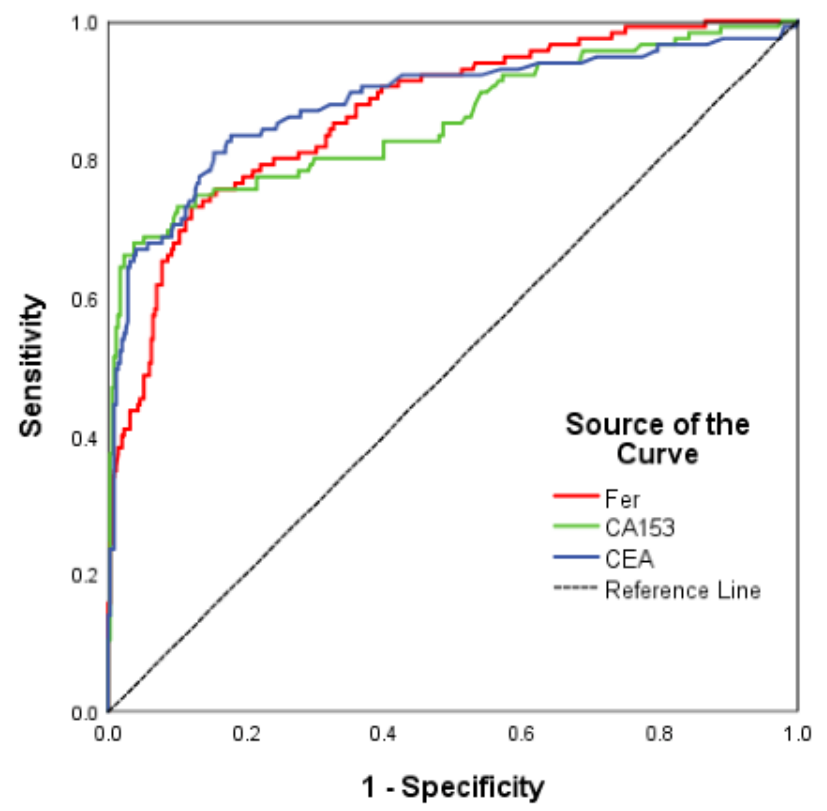


Figure 4

In different extent of tumor transfer to other metastatic sites, Receiver operating characteristic (ROC) curve analysis of different markers to predict the metastatic status. single distant metastasis; multiple distant metastasis.
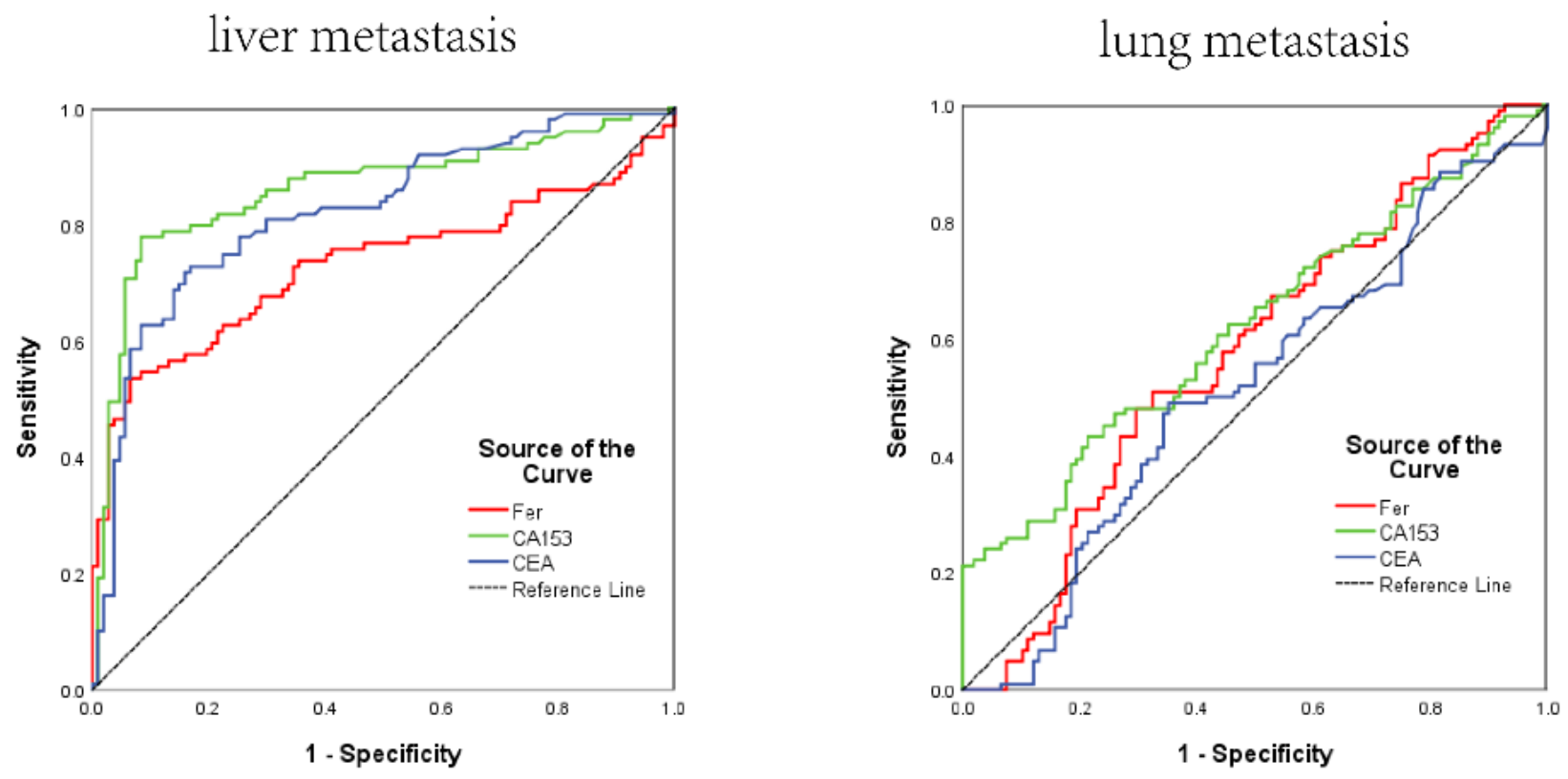

bone metastasis

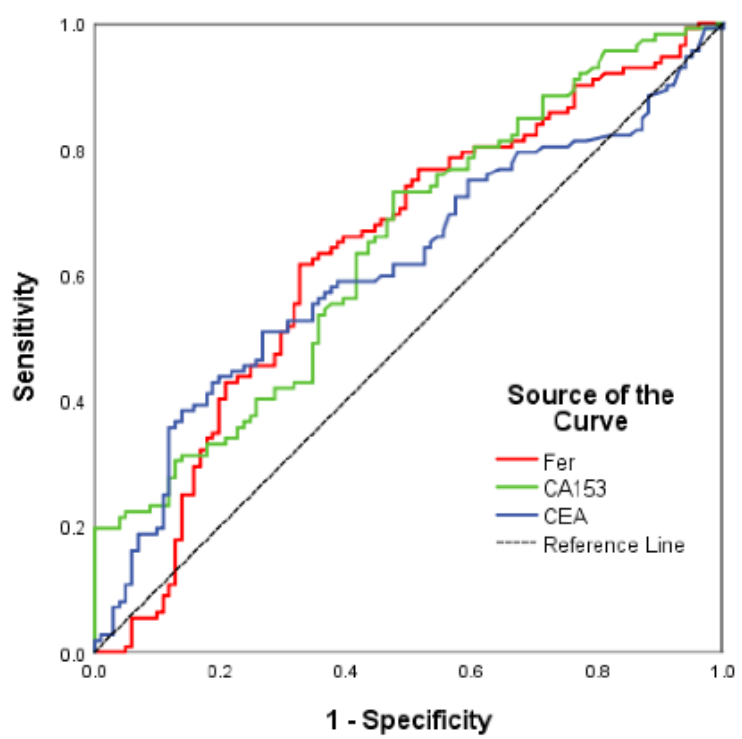

Figure 5

Receiver operating characteristic (ROC) curve analysis of sensitivity and specificity of three markers in predicting different metastatic sites. Liver metastasis; Lung metastasis; Bone metastasis.

\section{Supplementary Files}


This is a list of supplementary files associated with this preprint. Click to download.

- ROCAUC.docx

- metastasis.xlsx

- nonmetastasis.xlsx

- youdencutoff.docx 